\title{
Macroscopic Effects in Noncollinear High-Order Harmonic Generation
}

\author{
C. M. Heyl,'* P. Rudawski, F. Brizuela, S. N. Bengtsson, J. Mauritsson, and A. L'Huillier \\ Department of Physics, Lund University, P.O. Box 118, SE-221 00 Lund, Sweden
}

(Received 12 September 2013; published 7 April 2014)

\begin{abstract}
We study two-color high-order harmonic generation using an intense driving field and its weak second harmonic, crossed under a small angle in the focus. Employing sum- and difference-frequency generation processes, such a noncollinear scheme can be used to measure and control macroscopic phase matching effects by utilizing a geometrical phase mismatch component, which depends on the noncollinear angle. We further show how spatial phase effects in the generation volume are mapped out into the far field allowing a direct analogy with temporal carrier envelope effects in attosecond pulse generation.
\end{abstract}

DOI: 10.1103/PhysRevLett.112.143902

PACS numbers: 42.65.Ky, 42.65.Re

High-order harmonic generation (HHG) in gases using multicolor optical fields is becoming a common tool in attosecond science. Multicolor HHG has been implemented by mixing the fundamental field with waves at low harmonic [1-3] and at incommensurate [4,5] frequencies. Other schemes employ attosecond pulse trains mixed with the fundamental field [6] as well as synthesized light transients [7] for driving the HHG process. The control and flexibility brought about by using two or more driving fields is used, for example, to improve the efficiency of the generation process $[3,8]$, to monitor the single atom response $[9,10]$, and to implement gating techniques [11]. High-order wave mixing can further be beneficial for achieving phase matching [1,12-15].

Using multiple optical driving fields also allows for noncollinear geometries. This concept, used extensively in low-order frequency conversion processes such as optical parametric amplification, has barely been applied to attosecond science. In an early work, Birulin and co-workers [16] addressed theoretically the general concept of HHG in a noncollinear geometry. By mixing the fundamental and a weak second harmonic at an angle, Bertrand and co-workers [17] demonstrated the spatial separation of multiple extreme ultraviolet (XUV) beams corresponding to different sets of absorbed photon numbers from the two fields. A similar scheme was recently used [18] for reconstructing the spatiotemporal characteristics of the generated attosecond pulses. Other authors [19-21] concentrated on noncollinear sum-frequency generation (SFG) processes driven by two identical laser fields. Such a scheme allows for separation of the harmonics from the fundamental but suffers from phase matching problems.

Published by the American Physical Society under the terms of the Creative Commons Attribution 3.0 License. Further distribution of this work must maintain attribution to the author(s) and the published article's title, journal citation, and DOI.
In this Letter, we theoretically and experimentally examine the influence of noncollinear geometries in HHG, analyzing in detail the macroscopic aspects. We show for the first time, to our knowledge, how a weak noncollinear field can be used as a probe to monitor and control phase matching. We show that difference-frequency generation (DFG), where a photon (or several photons) is emitted in the conversion process, is in general more favorable than SFG [22], which becomes very inefficient with increasing noncollinear angle. This leads to the counterintuitive result that the XUV radiation is dominantly emitted outside the angle sector defined by the two driving (a)

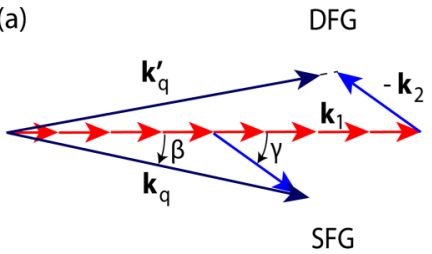

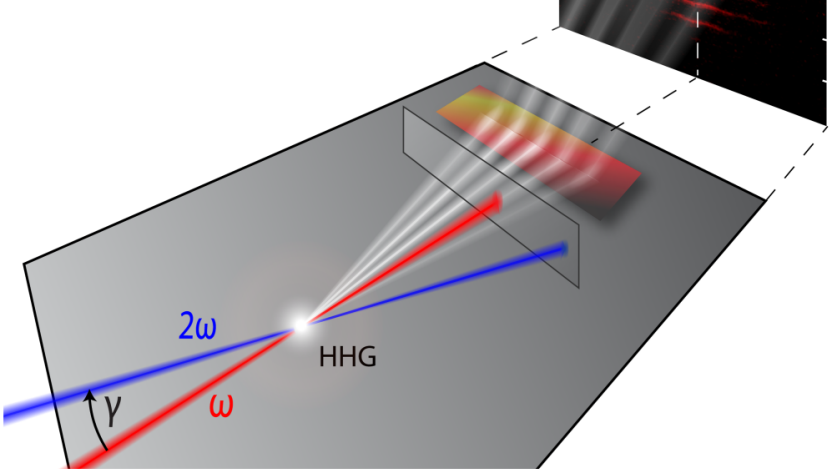

FIG. 1 (color online). (a) Wave vector representation of noncollinear wave mixing. (b) Schematic setup: a red $(800 \mathrm{~nm})$ and a blue $(400 \mathrm{~nm}$ ) laser beam are focused in a noncollinear geometry, resulting in the emission of spatially separated high-order harmonic beams. The depicted spectrum is a measured experimental microchannel plate image recorded at $\gamma \approx 2 \mathrm{mrad}$ and resampled for a linear energy scale. The noncollinear angle $(\gamma)$ is exaggerated in this illustration. 
fields. Our analysis is illustrated in an experiment where high-order harmonics are generated in argon using a high-energy $800 \mathrm{~nm}$ laser and its weak second harmonic, crossed under a noncollinear angle of a few mrad (Fig. 1). We identify two important regimes for noncollinear HHG: (i) the generation at noncollinear angles large enough to allow a direct spatial separation of all photon pathways leading to the same final energy [17] and (ii) the generation at smaller noncollinear angles leading to spatially resolved interferences between adjacent photon pathways. At large angles, our noncollinear generation scheme provides the functionality of an all-optical beam splitter, delivering multiple, angularly separated XUV beams. At small angles, the observed spatial interferences directly reflect the harmonic phase variation across the focal plane.

Noncollinear high-order harmonic generation illustrates beautifully the two fundamentally different but equivalent concepts of light, waves or photons. Both concepts are very useful for understanding the underlying principles and will therefore be used alternately in the discussion throughout this Letter. We begin by explaining the main features of noncollinear HHG resulting from the field distribution in the focal plane, followed by a more quantitative description including propagation effects.

In the photon picture, the combination of two laser fields with central frequencies $\omega_{1}$ and $\omega_{2}$ leads to radiation with frequency $\omega_{q}=m_{1} \omega_{1}+m_{2} \omega_{2}$, where $m_{1}$ and $m_{2}$ are integers. Without loss of generality $m_{1}$ can be chosen to be positive and $m_{2}$ positive or negative, accounting for sum- and difference-frequency generation processes, respectively. The emission angle $\beta$ of the generated radiation, measured from the propagation axis of the $\omega_{1}$ field, can be deduced through $k$-vector addition and defined by the unit vector of the generated field [23] $\hat{\mathbf{k}}_{q}=\left(m_{1} \mathbf{k}_{1}+m_{2} \mathbf{k}_{2}\right) /\left\|m_{1} \mathbf{k}_{1}+m_{2} \mathbf{k}_{2}\right\|$ [Fig. 1(a)]. Throughout the Letter we use small angle approximations.

We consider the case of a fundamental beam and its second harmonic, i.e., $\omega_{1}=\omega$ and $\omega_{2}=2 \omega$. The generated frequencies are $\omega_{q}=q \omega$ with $q=m_{1}+2 m_{2}$, where the net number of photons absorbed $m=m_{1}+m_{2}$ has to be odd in order to satisfy parity conservation. Denoting by $\gamma$ the angle between $\mathbf{k}_{1}$ and $\mathbf{k}_{2}, \beta$ can be approximated as

$$
\beta\left(q, m_{2}\right) \approx \frac{2 m_{2}}{q} \gamma, \quad m_{2}= \begin{cases} \pm 1,3, \cdots & \text { if } q \text { is even } \\ \pm 0,2, \cdots & \text { if } q \text { is odd }\end{cases}
$$

For sufficiently large $\gamma$, the number of $\omega_{2}$ photons defines a unique emission angle so that all driving photon combinations that lead to the same final energy are spatially separated in the far field. A pattern of harmonic beams can then be detected for each harmonic order, as illustrated in Fig. 2(a). For small noncollinear angles such that $\Delta \beta_{q}=\beta\left(q, m_{2}+2\right)-\beta\left(q, m_{2}\right)<\Theta_{q}$, where $\Theta_{q}$ is the divergence angle of a single harmonic beam, the

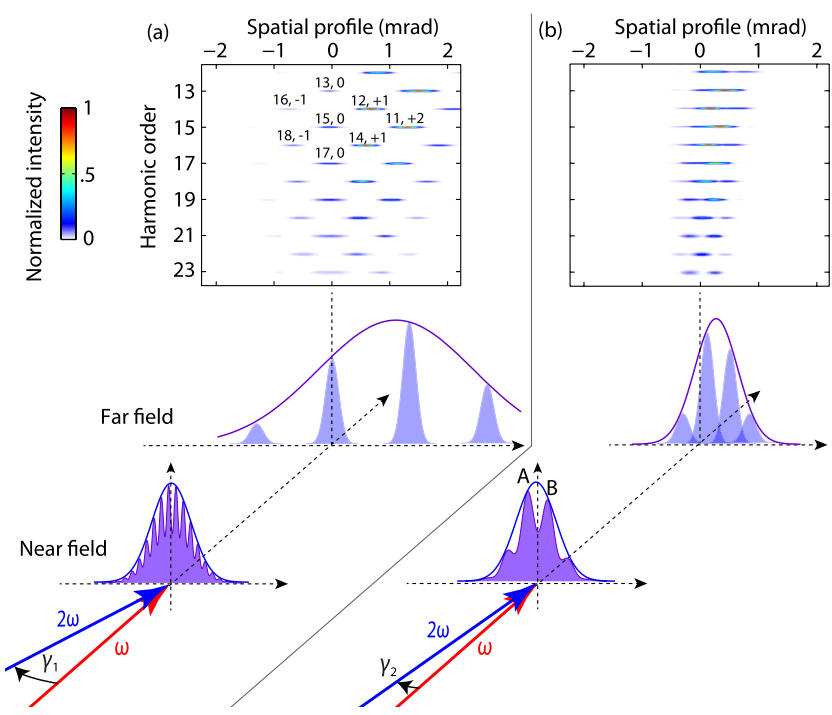

FIG. 2 (color online). Illustration of the interference concept: spatially resolved harmonic spectra as well as near- and far-field intensity distributions for a given harmonic order. (a) $\Delta \beta_{q}>\Theta_{q}$ and (b) $\Delta \beta_{q}<\Theta_{q}$. The spatial distribution of the high-order harmonic emission in the far field is defined by the interference grating in the focus representing multiple harmonic sources, determining intensity and phase of the emitted harmonics. The displayed far-field spectra were calculated using the strong field approximation, considering $\gamma=5 \mathrm{mrad}$ (a) and $\gamma=1.5 \mathrm{mrad}$ (b) with $I_{2 \omega} / I_{\omega}=0.05$ and $I_{\omega}=2 \times 10^{14} \mathrm{~W} / \mathrm{cm}^{2}$, where $I_{\nu}$ denotes the focus intensity at central frequency $\nu$.

harmonic beams with different $m_{2}$ orders can partly overlap spatially and interfere [Fig. 2(b)], as discussed in more detail later. The intensities of the two driving fields determine the probability for up-converting photons from one or the other driving field and define therefore the envelope position and width of the pattern in the far field. For the case of a weak $\omega_{2}$ field, harmonic emission occurs at small angles around $\mathbf{k}_{1}$ and only low $m_{2}$ orders can be expected. For a given harmonic order $q$, an $m_{2}$ order will be separated from the $\omega$ beam if $\gamma \geq q / 4 f_{\#} m_{2}$, where $f_{\#}=f / D$ is the $f$ number of the $\omega$ beam focused with a focal length $f$ and with an initial beam diameter $D$.

From a wave perspective, the generated far-field pattern can be understood as the interference of multiple harmonic sources. These sources are created by the interference of the two driving fields, as illustrated in Fig. 2. The modulation of amplitude and phase of the $\omega$ - $2 \omega$-driving field across the focal plane leads to an amplitude and phase modulation of the dipole oscillating at a given harmonic frequency. Note that the harmonic grating persists over the pulse duration while the intensity modulation at the fundamental frequencies is smeared out in time. All features of the far-field pattern mentioned above can be explained with this concept: the nonlinearity of the process determines the harmonic intensity 
(a) Collinear generation
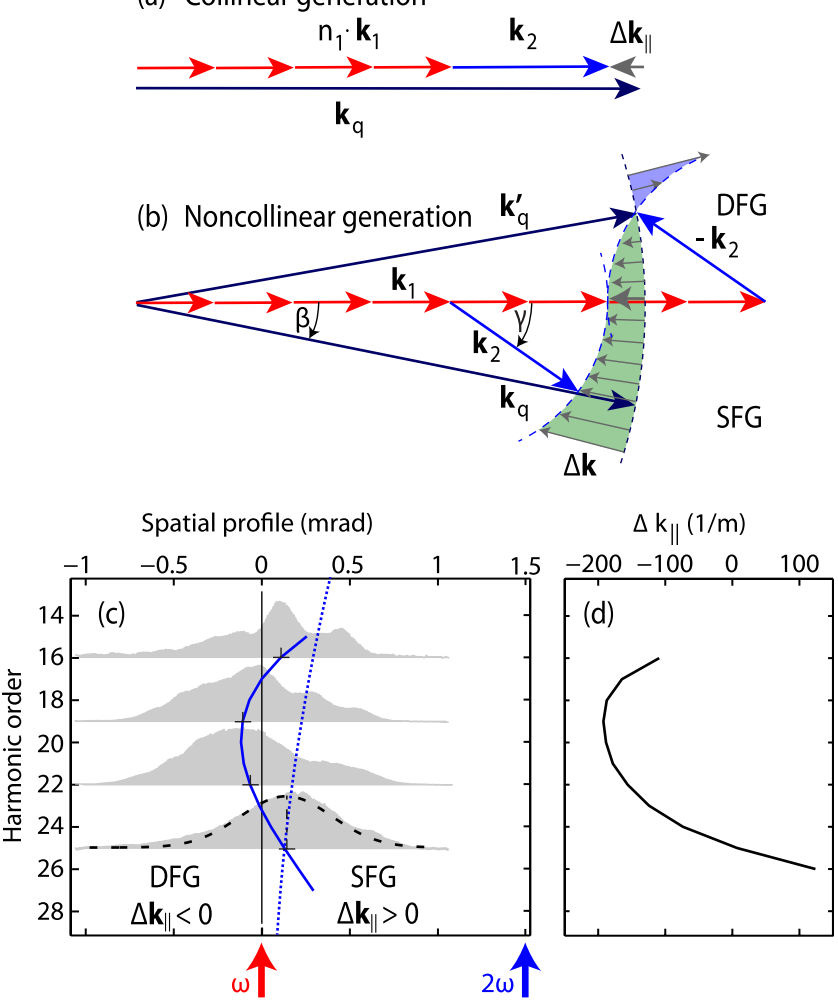

FIG. 3 (color online). (a),(b) Schematic phase matching scheme for noncollinear $\omega$-2 $\omega$ HHG. In conditions where a phase mismatch is present for a collinear generation geometry (here illustrated for a negative $\Delta \mathbf{k}_{\|}$on the optical axis), it is possible to find a corresponding noncollinear geometry at which the total phase mismatch is zero (e.g., considering DFG in the displayed scheme). The shaded areas indicate a negative (green) and positive (blue) $\Delta \mathbf{k}$. (c) Measured harmonic spatial profile averaged over the relative phase between the $\omega$ and $2 \omega$ fields for $\gamma \approx 1.5 \mathrm{mrad}$ : the solid blue line indicates the approximate center of the spatial profiles; the dotted blue line marks the calculated center of the harmonic beams in the absence of propagation effects. The two arrows mark the propagation directions of the $\omega$ and the $2 \omega$ beams. (d) Extracted wave vector mismatch $\Delta \mathbf{k}_{\|}$.

distribution in the focal plane and therefore the width of the envelope over the spatial distribution in the far field. The relative intensity of the two driving fields influences the phase of the source grid, which determines the position of the envelope in the far field.

Phase matching in HHG can be analyzed by adding the relevant wave vectors, as illustrated in Figs. 3(a) and 3(b). The total $k$-vector mismatch [24] for noncollinear HHG can be expressed as

$$
\begin{aligned}
\Delta \mathbf{k} & =\Delta \mathbf{k}_{\|}+\Delta \mathbf{k}_{<}, \\
\text {with } \quad \Delta \mathbf{k}_{<} & =m_{2} \gamma^{2} \underbrace{\left(\frac{2 m_{2}}{q}-1\right)}_{<0} \frac{\omega}{c} \hat{\mathbf{k}}_{q},
\end{aligned}
$$

where $\Delta \mathbf{k}_{\|}$is the $k$-vector mismatch corresponding to the collinear generation geometry [Fig. 3(a)] while $\Delta \mathbf{k}_{<}$is the geometrical offset added by the noncollinear geometry. Considering a certain $m_{2}$, phase matching $(\Delta \mathbf{k}=0)$ can be achieved by choosing the right angle $\gamma . \Delta \mathbf{k}_{<}$is positive or negative depending on the sign of $m_{2}$, i.e., on whether we consider DFG or SFG [Fig. 3(b)]. $\Delta \mathbf{k}_{\|}$can be written as a sum of four components [25]: the wave vector mismatch due to the geometrical Gouy phase, two components that arise from dispersion in the partially ionized medium, and a contribution due to the intensity-dependent dipole phase. A limiting factor is ionization, which is intrinsically required for HHG but strongly reduces the efficiency due to the negative phase mismatch induced by plasma dispersion. Additionally, the Gouy phase can lead to a significant negative phase mismatch especially in the case of a tight focus or high harmonic orders. In both cases, the resulting negative $\Delta \mathbf{k}_{\|}$can be compensated by a positive $\Delta \mathbf{k}_{<}$. In these conditions high harmonic emission driven by noncollinear DFG can dominate over noncollinear SFG. In the absence of propagation effects and for a weak perturbing $2 \omega$ field, the harmonic spatial profile is strongest at $\beta=0\left(m_{2}=0\right)$ [17]. If the far-field pattern is predominantly emitted outside the angle sector defined by the two driving fields (as in Fig. 1), DFG dominates, indicating that $\Delta \mathbf{k}_{\|}$is negative. If the emission maximum is located inside this angle sector, SFG dominates and $\Delta \mathbf{k}_{\|}$is positive. Not only the sign, but also the magnitude of $\Delta \mathbf{k}_{\|}$can be estimated as shown by the analysis of our experimental results presented below.

We performed experiments using a high-energy $40 \mathrm{fs} \mathrm{Ti}$ : sapphire laser system operating at a $10 \mathrm{~Hz}$ repetition rate [26]. The second harmonic field, with a pulse energy corresponding to $5 \%$ of the fundamental, was generated in one arm of a phase-stable Michelson-like interferometer and loosely focused together with the fundamental field into an argon gas cell $\left(f_{\#} \simeq 200\right)$. The interferometer allowed us to spatially displace the two beams so they could be focused with a small angle relative to each other. The generated harmonics were analyzed by an XUV spectrometer with an entrance slit and recorded by a microchannel plate detector. Our experimental conditions are chosen to strongly favor HHG emission from the short trajectory [26]. Due to the small angle, the beams corresponding to different $m_{2}$ orders interfere [case (b) in Fig. 2], and the spatial distribution in the far field depends on $\phi$, the relative phase between the $\omega$ and the $2 \omega$ fields. Figure 4(a) shows a single shot harmonic spectrum recorded with $\gamma \approx 1.5 \mathrm{mrad}$. Figure 3(c) presents the corresponding spatial intensity distributions for four harmonic orders, averaged over $\phi$. The solid blue line in Fig. 3(c) shows that the center of the far-field distribution, obtained by fitting Gaussian envelopes to the experimental data, varies with harmonic order. In order to deduce the effect of phase matching from the spatial distribution, the $\omega$-beam 

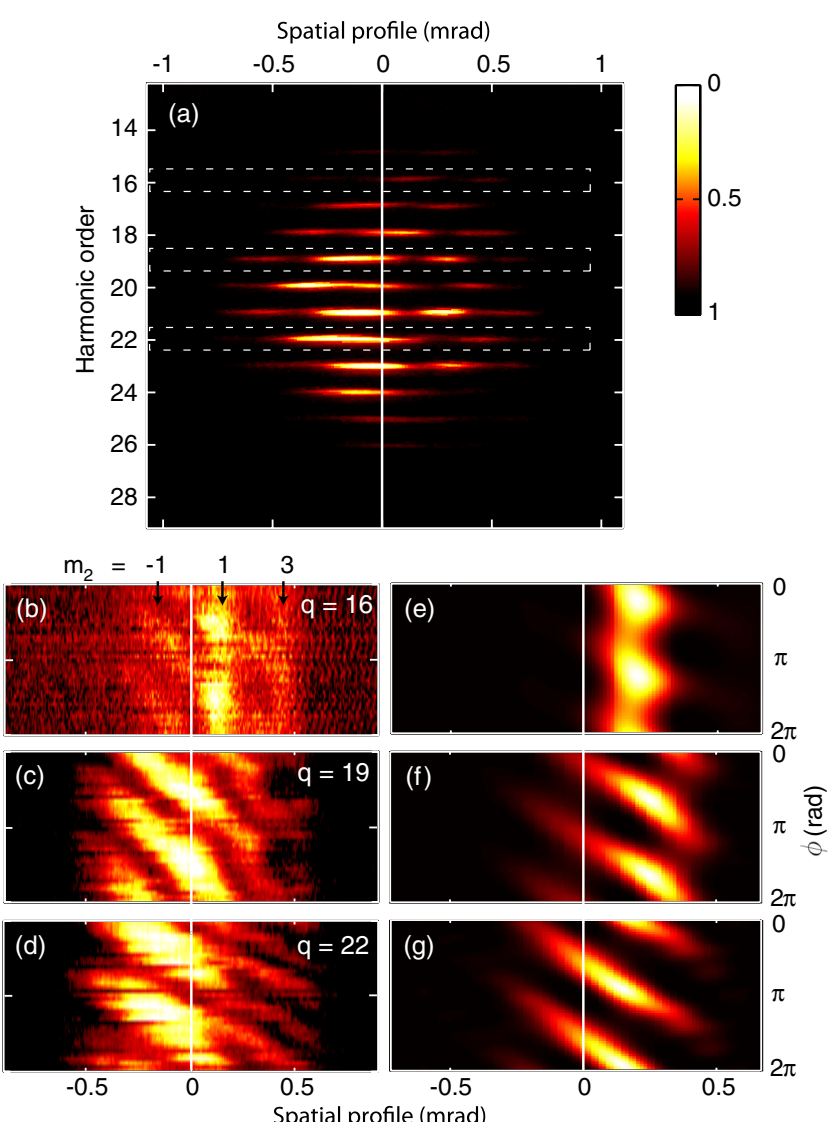

FIG. 4 (color online). (a) Single shot experimental harmonic spectrum generated in the same conditions as shown in Fig. 3(c). The dashed white lines indicate the region displayed spectrally integrated and normalized as a function of $\phi$ in (b)-(d). Corresponding SFA results are shown in (e)-(g). Note that the center of the spatial distribution is shifted compared to the experimentally measured patterns since propagation effects are not included in the simulations.

direction cannot be used as a reference, since the $2 \omega$-field intensity is not negligible in our experimental conditions. Instead we calculate this reference [dotted blue line in Fig. 3(c)] using numerical simulations based on the strong field approximation (SFA), accounting for the noncollinear overlap of the two driving fields but neglecting propagation effects (see the Supplemental Material [27]). Experimentally, we clearly observe a dominant contribution on the left side from the reference direction, for most harmonic orders, which we relate to a dominant DFG contribution in our experimental conditions. The offset between the experimental beam centers and the SFA results allows us to deduce an approximate value for the effective $\Delta \mathbf{k}_{\|}$as shown in Fig. 3(d) (see the Supplemental Material [27]).

Finally, we examine in more detail noncollinear HHG at small crossing angles and analyze the influence of interference effects on the harmonic spatial profile. Figures 4(b)-4(d) show the far-field profiles for harmonics 16,19 , and 22 as a function of $\phi$. The position of the spatial fringes varies periodically with $\phi$ and this variation becomes more pronounced as the harmonic order increases. This can be understood within the wave model: the harmonics generated at different source locations in the focal plane [e.g., $A$ and $B$ in Fig. 2(b)] interfere in the far field, and the interference pattern depends on their relative phase. The main contribution to this phase difference is due to the single atom response and can be expressed as $\Delta \varphi_{s}=\alpha_{q}\left(I_{A}-I_{B}\right)$, where $\alpha_{q} I$ denotes the harmonic dipole phase [28] at a corresponding driving field intensity $I$. When the phase $\phi$ is varied, the harmonic intensity grating in the focus moves across the focal plane and the difference between $I_{A}$ and $I_{B}$ changes. Consequently, the interference fringes in the far-field shift as clearly visible in Figs. 4(c) and 4(d). The slope reveals the dipole phase dependence on the intensity, which is small for low harmonic orders and increases with order, as expected for the short trajectory contribution [29]. Results from our SFA simulations, shown in Figs. 4(e)-4(g), reproduce fairly well the main features of the experimental interference patterns. The differences in width and shape of the spatial profiles (and consequently in the slope of the fringe pattern) can be explained by deviation from Gaussian optics including possible asymmetries of the fundamental beam shape in the focus as well as ionization effects leading to additional spatial broadening.

There is a clear analogy between the presented spatial phase effects and carrier envelope phase (CEP) dependent spectral fringes in HHG. Attosecond pulses are generated by a short fundamental pulse at a few instants in time, e.g., $t_{a}$ and $t_{b}$ at the corresponding driving field intensities $I_{a}, I_{b}$. The harmonic spectrum is a consequence of the interference between these pulses. Similarly to the spatial interference effect discussed above, the spectral interference pattern depends on the relative phase $\Delta \varphi_{t}=\alpha_{q}\left(I_{a}-I_{b}\right)$, which changes with CEP [30,31].

In summary, we have theoretically and experimentally investigated noncollinear HHG, focusing on the macroscopic aspects of the generation process. We have shown how a noncollinear second harmonic field can be used to probe phase matching by utilizing a geometrical phase mismatch component, introduced by the noncollinear geometry. Using difference-frequency generation processes, this component can be advantageous for phasematched generation in a partly ionized medium. We further identify different noncollinear angle regimes leading either to well separated harmonic beams or to spatial interference structures in the far-field spectrum. These interferences are the spatial analog of temporal interference structures in attosecond pulse generation with few cycle pulses.

This research was supported by the Marie Curie program ATTOFEL (ITN), the European Research Council (ALMA), the Swedish Research Council, the Swedish Foundation for Strategic Research, and the Knut and Alice Wallenberg Foundation. 
*Corresponding author. christoph.heyl@fysik.lth.se

[1] H. Eichmann, S. Meyer, K. Riepl, C. Momma, and B. Wellegehausen, Phys. Rev. A 50, R2834 (1994).

[2] S. Watanabe, K. Kondo, Y. Nabekawa, A. Sagisaka, and Y. Kobayashi, Phys. Rev. Lett. 73, 2692 (1994).

[3] F. Brizuela, C. M. Heyl, P. Rudawski, D. Kroon, L. Rading, J. M. Dahlström, J. Mauritsson, P. Johnsson, C. L. Arnold, and A. L'Huillier, Sci. Rep. 3, 1410 (2013).

[4] M. B. Gaarde, P. Antoine, A. Persson, B. Carré, A. L'Huillier, and C.-G. Wahlström, J. Phys. B 29, L163 (1996).

[5] T. Pfeifer, L. Gallmann, M. J. Abel, P. M. Nagel, D. M. Neumark, and S. R. Leone, Phys. Rev. Lett. 97, 163901 (2006).

[6] A Heinrich, W. Kornelis, M. P. Anscombe, C. P. Hauri, P. Schlup, J. Biegert, and U. Keller, J. Phys. B 39, S275 (2006).

[7] A. Wirth et al., Science 334, 195 (2011).

[8] I. J. Kim, C. Kim, H. Kim, G. Lee, Y. Lee, J. Park, D. Cho, and C. Nam, Phys. Rev. Lett. 94, 243901 (2005).

[9] N. Dudovich, O. Smirnova, J. Levesque, Y. Mairesse, M. Yu. Ivanov, D. M. Villeneuve, and P. B. Corkum, Nat. Phys. 2, 781 (2006).

[10] D. Shafir, H. Soifer, B. D. Bruner, M. Dagan, Y. Mairesse, S. Patchkovskii, M. Yu. Ivanov, O. Smirnova, and N. Dudovich, Nature (London) 485, 343 (2012).

[11] J. Mauritsson, P. Johnsson, E. Gustafsson, A. L'Huillier, K. Schafer, and M. Gaarde, Phys. Rev. Lett. 97, 013001 (2006).

[12] R. Hilbig and R. Wallenstein, Appl. Opt. 21, 913 (1982).

[13] P. Shkolnikov, A. Kaplan, and A. Lago, Opt. Lett. 18, 1700 (1993).

[14] S. Meyer, H. Eichmann, T. Menzel, S. Nolte, B. Wellegehausen, B. Chichkov, and C. Momma, Phys. Rev. Lett. 76, 3336 (1996).

[15] O. Cohen, T. Popmintchev, D. Gaudiosi, M. Murnane, and H. Kapteyn, Phys. Rev. Lett. 98, 043903 (2007).

[16] A. V. Birulin, V. T. Platonenko, and V. V. Strelkov, Quantum Electron. 26, 377 (1996).
[17] J. Bertrand, H. J. Wörner, H.-C. Bandulet, É. Bisson, M. Spanner, J.-C. Kieffer, D. M. Villeneuve, and P. B. Corkum, Phys. Rev. Lett. 106, 023001 (2011).

[18] K. Kim, C. Zhang, A. D. Shiner, S. E. Kirkwood, E. Frumker, G. Gariepy, A. Naumov, D. M. Villeneuve, and P. B. Corkum, Nat. Phys. 9, 159 (2013).

[19] S. V. Fomichev, P. Breger, B. Carré, P. Agostini, and D. F. Zaretsky, Laser Phys. 12, 383 (2002).

[20] J. Wu and H. Zeng, Opt. Lett. 32, 3315 (2007).

[21] A. Ozawa, A. Vernaleken, W. Schneider, I. Gotlibovych, Th. Udem, and T. W. Hänsch, Opt. Express 16, 6233 (2008).

[22] We emphasize that a geometrical phase mismatch introduced by the noncollinear generation geometry leads to enhanced DFG emission, which is different from earlier results that show dominating DFG emission due to dispersion effects in collinear geometries [15].

[23] Strictly speaking, this definition is an approximation, neglecting phase matching effects which can lead to a modified emission direction especially for large noncollinear angles and in the presence of radial field gradient vectors [32] caused e.g., by the intensity dependent dipole phase.

[24] We define the $k$-vector mismatch as the difference in $k$ vectors between fundamental and harmonic fields, e.g., $q \mathbf{k}_{1}-\mathbf{k}_{q}$.

[25] S. Kazamias, D. Douillet, F. Weihe, C. Valentin, A. Rousse, S. Sebban, G. Grillon, F. Augé, D. Hulin, and Ph. Balcou, Phys. Rev. Lett. 90, 193901-1 (2003).

[26] P. Rudawski et al., Rev. Sci. Instrum. 84, 073103 (2013).

[27] See Supplemental Material at http://link.aps.org/ supplemental/10.1103/PhysRevLett.112.143902 for a description of the numerical SFA model as well as for details on the extraction of the effective $k$-vector mismatch.

[28] M. Lewenstein, K. Kulander, K. Schafer, and P. Bucksbaum, Phys. Rev. A 51, 1495 (1995).

[29] K. Varjü et al., J. Mod. Opt. 52, 379 (2005).

[30] E. Mansten et al., Phys. Rev. Lett. 102, 083002 (2009).

[31] G. Sansone et al., Phys. Rev. A 80, 063837 (2009).

[32] P. Balcou, P. Salières, A. L'Huillier, and M. Lewenstein, Phys. Rev. A 55, 3204 (1997). 